Die Bildung und Erzengung eines Fettes aus. Zucker kann nicht gedacht werden ohne ein Austreten von Sauerstoff in der Form von Kohlensäure, ohne eine ähnliche Spallung des Zuckeratoms, wie sie in den Gährungsprocessen vor sich geht; sie kann nicht gedacht werden ohne eine entsprechende Verminderung seines Wasserstoffgehaltes, dessen Austreten aus dem Organismus in der Form von Wasser nur in Folge eines gleichzeitig eintretenden Verwesungsprocesses geschehen kann.

In diesen Bezieliungen hat der Uebergang des Zuckers in Felt die gröfste Aehnlichkeit mit dem Verwesungsprocesse der Holzfaser.

\title{
Ueber die Zusammensetzung und die medicinische Wirksamkeit des Chinoidins;
}

\section{von Demselben.}

Bei der Fabrikation des schwefelsauren Clinins bleibt zuletzl, wenn alles Krystallisirbare sich abgeschieden hat, eine dunkelgefärbte Mutterlauge von äufserst bitterem Geschmack zurijck, die bei Zusalz von kohlensauren Alkalien vollständig alle Bitterkeit verliert, indem sich ein gelblich weifser oder bräunlicher Niederschlag absetzt, der nach dem Auswaschen. mit Wasser in gelinder Wärme harzartig zusammenbackt. Aus den Versuchen Sertürner's, Thiele's, Buchholz d. J., Koch's und Anderer, ist es seil längerer Zeit bekannt, dafs diese harzähnliche Substanz basische Eigenschaften besitzt; sie neutralisirt die Sảuren vollkominen, aber die durch ihre Verbindung mit Säuren entstehenden Salze sind in keiner Weise zum Krystallisiren zu bringen. - Sertürner hielt diesen Körper, den er zuerst uns 
den Chininmulterlaugen isolirte, für eine eigenthümliche Basis, die neben dem Chinin und Cinchonin in den rothen und gelben Chinasorten enthalten ist; seinen Erfahrungen gemäls soll sie an Wirksamkeit das Chinin übertreffen, er gab ihr den Namen Chinoidin, und er nennt sie in seiner Zeitschrift (Ueber die nemeslen Fortschritte in der Chemie, Physik und Heilkunde, Bd. III, Heft 2, S. 269) einen wahren Ficbertödter. An manchen Orten ist das Chinoidin in Gebrauch gekommen und in den Preislisten aller Materialisten Deutschlands aufgenommen. - In den Mullerlaugen des scliwefelsauren Chinins, welche Henry und Delondre einer Untersuchung unterwarfen, sowie in einer Probe Chinoidin, welche Geiger untersuchte, entdeckten diese geschickten Chemiker einen Gehalt an Chinin und Cinchonin, begleitet von einem harzähnlichen Stoffe, von dem sie glaublen, dafs seine Gegenwart die Krystallisirbarkeit dieser beiden Basen in ihren schwefelsauren Salzen verhindert; dafs das Chinoidin je nach seinem Gehalte an Chinin eine ungleiche Wirksamkeit haben müsse, war hiernach nicht zu bezweifeln, und die Unsicherheit, in der man sich über diesen Gehalt befand, ist unstreitig der Grund gewesen, dafs viele Aerzte Bedenken trugen, sich des Chinoidin's als Arzneimittel zu bedienen. - Bei einer Durchreise durch Coblenz nahm ich mir aus der dortigen Materialhandlung Jobst und Comp. eine Probe Chinoidin mit, in der Absicht, es zur Darstellung des Chinoleïn's zu verwenden, jener organischen Basis, welche als Zersetzungsproduct des Chinin's von Gerhardt entdeckt wurde und neuerdings dadurch interessant geworden ist, dafs Prof. W. Hofmann dic Identität desselben mit dem im Steinkohlentheer vorkommenden Leukol erkannte. Enthielt es Chinin, so mufste es eine entsprechende Menge Chinoleïn liefern, und es liefs sich voraussetzen, dafs sich eine einfache Prüfung auf scinen Chiningehalt darauf begründen liefse. - Eine Probe von einigen Unzen Chinoidin, die 
mit slarker Kalilauge destillirt wurde, lieferte aher eine so üherraschend trofse Menge Chinoleïn, dafs man mit reinem Chinin nicht melır hätle erhalteu können, und diefs veranlafste zu einer näheren Untersuchung desselben, zu welcher ich mir, um nicht von einem vielleicht zufälligen Unstande getäuscht zu werden, gleichzeitig Proben von den Herren Hess, Leifsler und Ficdler in Mainz und Mettenheimer und Simon in Frankfurt, sowie von einer Materialhandlung in Hamburg kommen liefs. Diese verschiedenen Sorten Chinoidin erhiclt ich theils in unregelmaifsigen Massen, theils in viereckigen Tafeln von brauner oder schwarzbrauner Farbe; es wurde in der warmen Hand weich und biegsam, lief's sich aber in der Kàlte leicht pulvern, wobei es, wie das Chinin, aufserordentlich stark elektrisch wurde. Es zeigte sich völlig unlüslich in lialtem Wasser, in heifsem Wasser löste sich hingegen eine klcine Menge auf und ertheille demselben einen stark und rein bitteren Geschmack; in zwei Theilen Weingeist löste sich ein Theil von jeder dieser verschiedencen Proben Chinoidlu's vollkommen auf, diese Auflösung wird durch Zissalz von Wasser in harzähnlichen Flocken gefällt; sie lösten sich olne Rückstand in sehr verdüinnten Mineralsäuren, sowie in den meisten organischen Säuren, welche durch eine hinreichende Menge Clinoidin vollständig neutralisirt wurden. Aus diesen Auflösungen wurde das Chinoidin durch Ammoniak und kohlensaure Alkalien vollständig niedergeschlagen. Schüttelte man die Flüssigkeit, worin sich der suspendirte Chinoidinniederschlag befand, wiederholt mit ihrem gleichen Volum Aether, so löste sich der Niedersclllag bis auf einen höchst unbedeutenden schwarzbraunen Rückstand in dem Aether auf, und diese ätherische Lösung hinterliefs es nach dem Verdampfen mit allen seinen früheren Eigenschaften. Die Chinoidinsalze werden durch Gerbsäure gefällt, durch Platinchlorid erhält man in der salzsauren Lösung einen gelben, dem Chininplatinsalz vollkomunen 
gleichen Nicderschlag. Dafs das Chinoidin seiner ganzen Masse nach aus einem organischen Alkali besteht, geht noch daraus hervor, dals es sich beim Erwärmen mit einer Auflösung von schwefelsaurem Kupferoxyd unter Abscheidung des Kupferoxyds vollständig auflöst. Es giebt kein Harz, oder keine den Harzen älnnliche Materie, der diese Eigenschaft zukommt. Von Chinin oder Cinchonin liefsen sich darin nur Spuren nachweisen. Was den chemischen Character des Chinoidins betrifft, so läfst sich, wie ich glaube, nach dem Vorhergehenden kein Zweifel darüber hegen, die Elementaranalyse hat zuletzt ergeben, dafs es identisch in seiner Zusammensetzung mit dem Chinin ist und nicht blofs die nämlichen Elemente in gleichem Verhältnisse wie dieses enthält, sondern auch das nämliche Atomgewicht besitzt. Zu den folgenden Analysen wurde das Chinoidin entweder in dem Zustande angewendet, in welchem es aus der obenerwähnten Lösung in Aether nach dem Verdampfen desselben zurückblieb, oder es wurde dazu der Niederschlag benutzt, den man aus der Auflösung des rohen Chinoidin's in schwefelsaurem Kupferoxyde nach der Entfernung des Kupfers durch Schwefelwasserstoff mit einem Alkali erhielt. Aus der salzsauren Lösung. des nach einer dieser Methoden gereinigten Chinoidin's mit Platinchlorid erhielt man das zur Analyse verwendete Chinoidin-Platinsalz.

I. Chinoidin aus Mainz; 0,490 Grm. lieferten 1,3204 Kohlensäure und 0,3395 Wasser.

II. Chinoidin aus Frankfurt; 0,618 Grm. lieferten 1,6575 Kollensäure und 0,4250 Wasser.

III. Chinoidin aus Coblenz; 0,3475 Grm. lieferten 0,9475 Kohlensäure und 0,2375 Wasser.

Die Stickstoffbestimmung nach der Methode von Varrentrapp und Will gab folgendes Resultat :

0,515 Grm. Chinoıdin gaben 0,289 Grm. Platin; entsprechend 8,04 pC. Stickstoff. 
352 Liebig, über die Zusammensetzung und die medicinische

0,617 Grm. Chinoidin gaben 0,401 Grm. Platin $=9,54 \mathrm{pC}$. Stickstoff.

Nach dem Millel beider Analysen enthält das Chinoidin 8,79 pC. Stickstoff.

Nach diesen Kohlenstoff -, Wasserstoff - und Stickstoffbestimmungen enthàlt das Chinoidin :

I. II. III.

$\begin{array}{lrrr}\text { Kohlensloff *) } & \mathbf{7 3 , 4 9} & \mathbf{7 3 , 1 4} & \mathbf{7 4 , 3 3} \\ \text { Wasserstolr } & \mathbf{7 , 6 9} & \mathbf{7 , 6 4} & \mathbf{7 , 5 7} \\ \text { Stickstoff } & \mathbf{8 , 7 9} & 8,79 & \mathbf{8 , 7 9} .\end{array}$

Analyse des Chinoidinplatinsalzes.

I. 0,6663 Grm. Platinsalz lieferten 0,1755 Platin.

$0,8700 \gg$ dessclben Salzes gaben 1,03:19 Kohlensäurc und 0,303 Wasser.

II. 0,881 Grm. Chinoidinplatinsalz hinterliefsen 0,224 Platin.

III. $1,0668 \%$ " $" 0,2715$,

Nach diesen Bestimmungen enthalten 100 Theile Chinoidinplatinchlorid :

\begin{tabular}{lrcc} 
& \multicolumn{1}{c}{ I. } & II. & III. \\
Kohlenstoff & 32,44 & $\eta$ & $"$ \\
Wasserstoff & 3,86 & $\pi$ & 7 \\
Platin & 26,33 & 26,32 & $26,45$.
\end{tabular}

Vergleicht man den Platin, den Kohlenstoff und Wasserstoffgehalt des Chinoidinplatinsalzes und den Kohlen- und Wasserstoffgehalt des reinen Chinoidins mit der Zusummensetzung des Chinins und des entsprechenden Chininplatinsalzes, so ergiebt sich von selbst, dafs Chinin und Chinoidin für sich und in ihrem Platinsalz einerlei Zusammensetzung besitzen. - Nach der Formel $\begin{array}{lllll}\mathrm{C}_{20} & \mathrm{H}_{12} & \mathrm{NO}_{2} & \text { enthält das Chinin : }\end{array}$

*) Kohlenstoff $=\mathbf{7 5}$. 


\section{Kohlensloft $\mathbf{7 4 , 3 3}$ \\ Wasserstoff 7,57.}

Das Chininplatinsalz enthält nach der Formel :

$\mathrm{Cl} \mathrm{H}, \mathrm{C}_{20} \mathrm{H}_{12} \mathrm{~N} \mathrm{O}_{2}+\mathrm{Cl} \mathrm{P}$

Kollenstoin 32,38

Wasserstoff 3,53

Platin 26,83.

Das Chinoidin steht nach diesen Versuchen zu dem Chinin in einer ähnlichen Beziehung, wie der krystallisirbare Zucker zu dem unkrystallisirbaren, beide haben einerlei Zusammensetzung und einerlei Atomgewicht, sie liefern durch ihre Zersetzung gleiche Producte und sind nur der For'm nach von einander verschieden. Ich halte dieses Resultat für wichtig genug, un die Aufmerksamkeit der praktischen Aerzte darauf zu lenken, besonders in dem gegenwärtigen Augenblick, wo der hohe Preis des schwefelsauren Chimins (das Pfund schwefelsaures Chinin lio tet $86 \mathrm{fl}$.) seine Anwendung in einer Menge von Fällen, namentlich in der Armenpraxis, beschränkt. Nach den Erfahrungen, die bereits über das Chinoidin vorliegen, und die lange nicht so bekannt sind, als sie es verdienen, kann man mit Bestimmtheit behaupten, dafs ein Pfund Chinoidin, welches nicht über zwöll Gulden kostet, densclben Wirkungswerth und medicinischen Effuct besilzl, als ein Pfund schwefelsaures Chinin, welches einen siebenmal höheren Preis besitzt; so kann es lieine Frage seyn, dafs der Organismus keinen Unterschied kennt zwischen einem und demselben Stoffe im antorphem oder krystallisirtem Zustande, und eine in ibren Wirkungen so sichere und kostbare Arznei verdient ohne Zweifel die sorgfältigste Beachtung. Für den Handel ist es natürich von grofsem Werth, wern aus dem blofsen Ansehen eines Arzneimittels, wie aus dem schön krystallisirten Chininsalze, sein Gehalt und seine Reinheit, oder seine Wirksamkeit beurtheilt werden kann; diefs ist bel denı Chinoidin nichı der Fall, was die Gleichgültigkeil erklürt, 
die man in Allgemeinen dagegen wahrnimnt, allein das Castorrum, las Opium und viele andere der wirksamsten Mittel, besibzen ganz dic Beschaffenheit des Chinoillins, sie bedürfen vor ilurer Verwendung einer Prüfung in Beziehung auf ihre Gïtc und Reinheit, und die Nothwendigkeit oder Nützlichkeit derselben schliefst ihre Anwendung nicht aus. Es giebt übrigens kaum ein Arzneimittel, bei welchem die Prüfungsmethoden so einfach sind, wie bei den organischen Basen; ihre vollkommene Löslichkeit in verdiimnter Säure, in Alkohol oder Aether, unterseheidet sie von ailen andern dem äufseren Ansehen nach ähnlichen orgumischen Sulsstanzen. Wenn eine Probe Chinoidin sich in diesen drei Flüssigkeiten löst und die Auflösung in einer verdünnten Säure mil Ammoniak niedergeschlagen, eine dem Gewicht des Aufgelösten gleiche Menge Niederschlag giebt, so kanil man über seine Reinheit sicher seyn. Es sind mir übrigens Proben von Chinoidin zu Gesicht gekommen, die aus nichts anderm bestanden, als aus der zur Trockne eingedampften Multerlauge des schwefelsauren Chinins, oder wenn man will, aus schwefelsaurem Chinoidin. Diese Art von Chinoidin löst sich unvollständig in Wasser zu einer dunkelhramen Flïssigkeit auf, aus der man iibrigens nach der Behmndlung mil ausgewaschener Knochenkolle durch Zusalz von Ammoniak ein reines Chinoidin erlalten kann.

Ich halle es für angemessen, und glaube vielen Aerzlen cinen wahren Dienst zu leisten, wenn ich dieser Notiz das Urtheil eincs sehr erfahrenten Arztes, des Hrn. Geheimen Rathes Dr. Natorp in Berlin, welcher seit langem schon die Wirksamkeit des Chinoidins erkannt hat, folgen lasse; cs ist aus einem Briefe enluommen, den derselbe abzudrucken mir bereitwillig, crlaubt hat.

Aus einem Briefe des Geh. Sanitätsrathes Dr. Natorp zu Berlin an Dr. Bensch, Assistenten an dem chemiscluen Laboratorium, vom 18. Angust 1844. "Du schuruilst mir, ter Hr. 
Prof. Liebig wünsclıe von mir zu wissen, ob das Chinoidin noch jetzt gegen Wechselfieiver in Berlin grbraucht werde, und wünschest, dafs ich Dir meine Erfahrungen darüler mitheilen inöge.

„Um die erste Frage, ob es hier noch viel gebraucht werde, zu beantworten, mulste ich bei den Apothchern Nachfrage hallen, und wählte ich hicrzu die Apothekercoulerenz, wo ich alle zusainmen fand. Erst gestern fand diese Statt und hörte ich, dals das Chinoidin, namentlich als Tinct. Chinoid., noch inmer häufig verordnet werde, nicht blofs als Mittel gegen Wechselfieber, sonderu aucl: als Roborans.

„Was meine Erfahrungen über dieses vortreffliche Mittel betriffl, so bediene ich mich gegen Wechselfieber desselben ausschliefslich, wo es mir darauf ankommt, dieses Leiden zu heben, und kamn ich aus langjähriger Erfahrung bchaupten, dafs es mich nio im Stich gelassen hat. Ich habe bei diesem Mittel den Vortheil, mil Gewifsheit bestinmen zu kömen, ob der nächste Anfall ausbleiben solle, was bei allen anderen Chinapräparaten nicht so bestimmt ist, und verhütet kein anderes Mittel die Recidive so wie dieses. Nie habe ich bei den 'lausenden von Fällen, wo ich es angewandt habe, irgend eine nachlheilige Wirkung von demselben gesehen, nie eine Nachkrankheit.

תIch glaube ziemlich der erste gewesen zu seyn, der das Mittel hier in Berlin in Anwendung gezogen hat. Der verstorbene Apotheker Riedel, welcher grofse Quantitäten China bearbeitete, behielt diesen Stofl' als Rückstand und konnte ihn nicht weiter reinigen, glaubte aber, dafs er noch als Medicament gebraucht werden könnte und theilte mir eine Anzahl Josen davon mit, um Versuche damit in der Armempraxis anzustellen. Eine damals herrschende Fieberepidemie gal) hierzu Gelegenheit, und war ich von der Wirkung überrascht. $\Lambda$ uch der Regimentsarat Dr. Schilling hatle bei scinen Versuchen dieselben günsligen 
Resullate. Da auch in der Umgegend von Berlin auf den Dörfern viele Fiolerkinalie vorkamen, so verlangten einige Gutsbesitzer der Limgegend, deren Arzt ich bin, Mitlel gegen die Fieber; ich verschrieb die Tinct. Chinoidinae, und alle vertilgten bald die Krankheit auf ihren Gütern, ja der Ruf dieser Kuren verbreilete sich in der Umgegend so, dafs die Landleute zehn Meilen weit nach Fr. und Br. kamen, un sich die Fiebertropfon zu holen. Hr. v. Tr., welclser grofse Güler hei Warschau hat, sandte das Hittel während einer heftigen Weviselfieberepidemie dort in grofsen Quantitäten hin, wo es mit dern günstigsten Erfolg gebraucht wurde.

"Die Art, wie ich das Mittel verordne, ist folgende :

R. Tinct. Chinoidinae Unc. 1

Acid. Halleri Dr. 1

Aq. Menth. piper. vinos. Unc. 3

M D S.

„Bei eintägigen Fiebern beim Eintritt des Schweilses 1 Theelöffel voll alle Stunden.

"Bei dreilägigen Fiebern beim Eintrilt des Schweifses 1 'Theelöffel voll alle zwei Slunden.

"Bei viertigigen Fiebern beim Eintrilt des Schweifses 1 Theelöffel voll alle drei Stunden.

¿So wie das Fieber weggeblieben ist, was nicht leicht felılt, ich könnte wohl sagen, nie fehlt, Abends und Morgens einen Theelöffel, bis die Aussicht auf Recidive getilgt ist. Auch in den letzten Monaten hat sich das Mittel wie früher bewährt, wem gleich die Fieber nur sporadisch vorkamen.4 